\title{
20. Description d'un nouveau genre d'Hirudinée de la famille des Glossiphonides, Oligoclepsis tukubana n. g. n. sp.
}

\author{
Par Asajiro OKA, M.I.A. \\ Tokyo.
}

(Comm. Feb. 12. 1935.)

Depuis longtemps je n'ai eu entre les mains aucune espèce nouvelle d'Hirudinée d'eau douce indigène, de sorte que j'ai été amené à croire que tout y soit déjà connu. Or, le 26 novembre dernier (1934) M. S. Hatakeyama m'adressait trois Clepsines, recueillies par lui depuis peu au mont Tukuba, lesquelles présentaient des caractères tout à fait exceptionnels, nécessitant la création d'un nouveau genre pour ces Sangsues.

\section{Oligoclepsis tukubana n. g. n. sp.}

La forme du corps est exactemeut celle d'une Placobdella, c'est-àdire, ovoïde allongée, à grosse extrémité tournée en arrière, aplatie. Tous les trois exemplaires sont à peu près de la même taille, mesurant $12 \mathrm{~mm}$ de long et $6 \mathrm{~mm}$ de large au maximum. Le dos, qui est légèrement convexe, est orné d'une multitude de papilles rangées en lignes transversales; le ventre, au contraire, est parfaitement lisse. La couleur est d'un gris uniforme, sans bandes ni taches, un peu plus claire à la face ventrale. L'extrémité céphalique n'est nullement élargie, comme chez l'Hemiclepsis. La ventouse postérieure est petite, circulaire et cachée pour la plupart sous le ventre.

Ce qui distingue nettement ce nouveau genre de tous les autres dejà connus, c'est que le somite est formé, à la face dorsale, par deux anneaux sensiblement égaux, tandis qu'il comprend, à la face ventrale, trois anneaux, dont les deux premiers sont séparés l'un de l'autre par un sillon beaucoup moins profond que celui qui le suit. De plus, les rangées transversales de papilles dorsales occupent, sur l'anneau postérieur de chaque somite, la zone moyenne, mais sur l'anneau antérieur, elles sont placées le long du sillon interannulaire, de quoi il résulte que les rangées forment des groupes de deux, correspondant aux somites qui les portent. Les papilles sont d'ailleurs de taille variable, et leur arrangement est loin d'être très régulier. 
Le corps, en avant de la ventouse postérieure, est constituté, comme à l'ordinaire, de 27 somites. Les somites I à VII sont parfaitement uniannulaires, on n'y voit aucune trace de sillon interannulaire. Le somite VIII montre un sillon très peu profond, on peut dire que ce somite est intermédiaire entre uni-annulé et bi-annulé. Mais à partir du somite IX, le sillon interannulaire devient distinct, et cet état se continue jusqu'au somite XXII. Le somite XXIII est de nouveau quasi biannulé, le sillon interannulaire étant sensiblement moins profond que les précédents. Les quatre derniers somites, XXIV à XXVII, sont formés chacun d'un seul anneau. A la face ventrale, dans la région moyenne du corps, le somite comprend trois anneaux, mais vers les deux extrémités, on trouve la même réduction graduelle des sillons interannulaires, d'abord de l'antérieur, puis du postérieur.

La ventouse orale occupe la face ventrale des somites I à IV ;

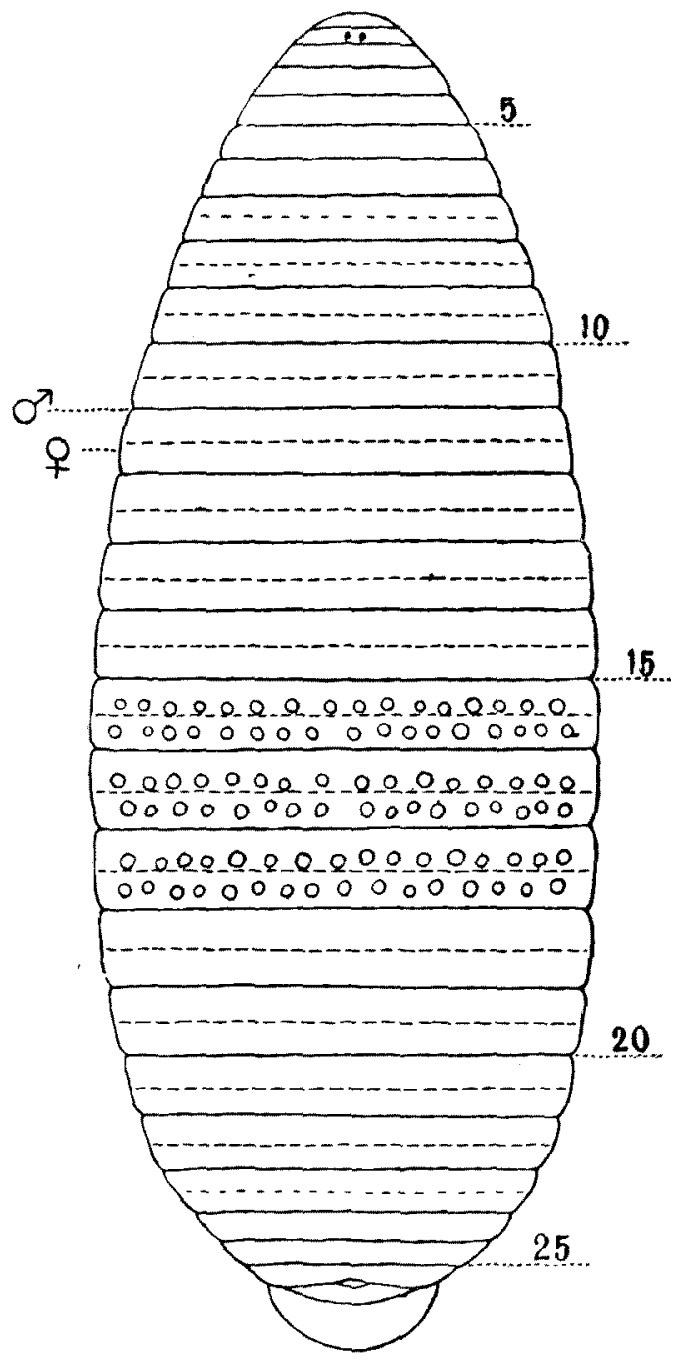

Oligoclepsis tukubana, grossie.

Les chiffres indiquent le numéro d'ordre des somites. La disposition des grosses papilles dorsales est indiquée sur les somites XVI à XVIII. elle est relativement très petite. L'orifice buccal se trouve à peu de distance du bord de la lèvre antérieure, en avant du centre de la ventouse.

Il y a une paire d'yeux, situés dans le somite II, très rapprochés l'un de l'autre. Ils sont noirs et très apparents.

L'orifice génital mâle est situé dans l'interstice des somites XI et XII. L'orifice femelle débouche dans le sillon séparant les deux anneaux, grand et petit, qui composent le somite XII. L'un et l'autre sont petits et n'offrent rien de particulier. Quant au clitellum, aucun des 
exemplaires ne le présentai avec assez de netteté pour qu'il fût possible d'en fixer exactement les limites.

L'anus s'ouvre entre le dernier et l'avant-dernier anneau.

Les animaux se trouvaient sous des pierres dans de petits ruisseaux. L'un d'entre eux portait sous le ventre environ trente petits, qui montraient déjà nettement les deux yeux noirs.

Comme on le voit aisément de la description ci-dessus, le nouveau genre est unique dans le fait, que le somite est formé de deux anneaux à peu près égaux à la face dorsale, tandis qu'il se divise en trois anneaux à la face ventrale. Cependant, la position des papilles dorsales sur la moitié postérieure du premier anneau de chaque somite semble indiquer que celui-ci correspond en réalité aux deux premiers anneaux d'un somite triméridé, tel que le possèdent ordinairement les Sangsues de la famille des Glossiphonides. 